\title{
Predictive value of perfusion weighted imaging for early new lesions after stroke patients receive endovascular treatment
}

\author{
Liang Jiang ${ }^{1 \#}$, Zhongping Ai ${ }^{1 \#}$, Wen Geng ${ }^{1}$, Huiyou Chen ${ }^{1}$, Boxiang Zhao ${ }^{2}$, Haobo Su ${ }^{2}$, Xindao Yin ${ }^{1}$, \\ Yu-Chen Chen ${ }^{1}$
}

${ }^{1}$ Department of Radiology, Nanjing First Hospital, Nanjing Medical University, Nanjing, China; ${ }^{2}$ Department of Intervention, Nanjing First Hospital, Nanjing Medical University, Nanjing, China

Contributions: (I) Conception and design: L Jiang, Z Ai; (II) Administrative support: X Yin, YC Chen; (III) Provision of study materials or patients: B Zhao, H Su; (IV) Collection and assembly of data: W Geng, H Chen; (V) Data analysis and interpretation: L Jiang, Z Ai; (VI) Manuscript writing: All authors; (VII) Final approval of manuscript: All authors.

\#These authors contribute equally to this work.

Correspondence to: Xindao Yin, MD, PhD; Yu-Chen Chen, MD, PhD. Department of Radiology, Nanjing First Hospital, Nanjing Medical University, No.68, Changle Road, Nanjing 210006, China. Email: y.163yy@163.com; chenyuchen1989@126.com.

Background: Previous studies have focused on early new lesion-associated factors, but the differences in the perfusion status between the at-risk hypoperfusion areas with new lesions and the other hypoperfusion areas in stroke patients before thrombectomy is not clear. We investigated the value of perfusion-weighted imaging (PWI) in predicting early new lesions in patients after stroke.

Methods: Fifty-five acute stroke patients who underwent diffusion-weighted imaging (DWI) and PWI before and after thrombectomy within $24 \mathrm{~h}$ were eligible. The PWI parameters of the core infarct areas (high signal tissue on the DWI), the at-risk hypoperfusion areas (hypoperfusion area with new lesions at followup PWI) and the other hypoperfusion areas of patients with new lesions were collected. Statistical analysis was performed to predict new lesions after stroke. The differences in the PWI parameters of the core infarct areas, the at-risk hypoperfusion areas and the other hypoperfusion areas were compared. Receiver operating characteristic (ROC) curve analysis was used to assess the predictive value of the PWI parameters $(\mathrm{P}<0.05)$ for the occurrence of new lesions in patients with acute stroke after thrombectomy.

Results: Fifty-five stroke patients were analyzed, including forty patients $(72.73 \%)$ with new lesions and fifteen patients $(27.27 \%)$ without new lesions. Acute stroke patients with new lesions had a longer mean transit time (MTT) and time to peak (TTP) in the at-risk hypoperfusion areas $(11.95 \pm 3.29 ; 38.30 \pm 11.39)$ than in the other hypoperfusion areas $(8.68 \pm 2.08 ; 29.76 \pm 6.86)$, both of which were significantly different $(\mathrm{P}<0.0001 ; \mathrm{P}<0.0001$, respectively). The ROC analysis showed that the sensitivity and specificity of MTT for predicting the occurrence of new lesions after stroke were $70.00 \%$ and $87.50 \%$, respectively; the sensitivity and specificity of TTP were $70.00 \%$ and $80.00 \%$, respectively.

Conclusions: MTT and TTP may be useful in predicting early new lesions in acute stroke patients after thrombectomy.

Keywords: Stroke; magnetic resonance imaging (MRI); perfusion-weighted imaging (PWI); new lesions

Submitted Jan 01, 2021. Accepted for publication Apr 09, 2021.

doi: 10.21037/qims-21-1

View this article at: http://dx.doi.org/10.21037/qims-21-1 


\section{Introduction}

The evolution of stroke often occurs after acute stroke following reperfusion therapy (1). Stroke evolution is a complex process and can be divided into at least the following two types: development of new lesions and enlargement of the infarct compared to the initial lesions. Early new ischemic lesions are common in patients with acute ischemic stroke. Serial magnetic resonance imaging (MRI) studies have found that new ischemic lesions appeared on diffusion-weighted imaging (DWI) in approximately $30 \%$ of patients within 1 week after symptom onset (2). Most of the patients with new ischemic lesions lack obvious new neurological symptoms (appear clinically silent) $(3,4)$. However, new ischemic lesions, regardless of whether they are silent or symptomatic, increase the risk of disability and mortality (5). New lesions may provide a useful surrogate endpoint in clinical trials evaluating stroke prevention therapies, with the reduction in the lesion recurrence rate upon pharmacologic intervention over the initial weeks. Therefore, the identification of high-risk patients for new ischemic lesions and the exploration of effective secondary stroke prevention methods are very important.

Thus far, the pathophysiology of these new lesions remains unclear. New lesions may result from incomplete clot dissolution and distal embolization in patients who underwent vessel recanalization therapy $(6,7)$, or new lesions may represent de novo lesions (e.g., new lesions located remotely from the area of initial hypoperfusion) that possibly originate from a proximal source, such as the aortic arch or heart. In previous studies, the development of new lesions was found to be associated with multiple ischemic lesions $(3,8,9)$. Among the different stroke subtypes, large-artery atherosclerosis was thought to be the type most frequently associated with new lesions $(8,10)$. In addition, thrombolytic therapy and vessel recanalization have also been confirmed to be associated with new lesions $(8,11)$. Most recent studies have focused on the related factors influencing the formation new lesions (12-15), but it is not clear if the perfusion status in the at-risk hypoperfusion areas with new lesions differs from that of other hypoperfusion areas in patients before thrombectomy.

Semi-quantitative perfusion maps of cerebral blood flow (CBF), cerebral blood volume (CBV), time to peak (TTP), mean transit time (MTT) could be generated from Perfusion MRI. Our hypothesis was that the new lesions in stroke patients with large vessel [middle cerebral artery (MCA) or/and internal carotid artery (ICA)] occlusion
(LVO) would show different perfusion statuses compared to the initial lesions or hypoperfusion areas before thrombectomy. In the present study, we analyzed the extent of hypoperfusion using perfusion-weighted imaging (PWI) to compare the difference between the hypoperfusion areas with new lesions and the other hypoperfusion areas in stroke patients with LVO before endovascular therapy. In addition, we also assessed whether the PWI parameters can predict the formation of new lesions in stroke patients with LVO after endovascular therapy.

\section{Methods}

\section{Subjects and clinical data}

The study was conducted in accordance with the Declaration of Helsinki (as revised in 2013). The hospital review board of Nanjing Medical University approved the study protocol. Acute stroke patients who were admitted to the Nanjing First Hospital from January 2017 to November 2018 within $6 \mathrm{~h}$ of the onset of ischemic symptoms were evaluated retrospectively. Acute stroke was defined as acute clinical vascular syndrome with evidence of a cerebral infarction on DWI. Patients who met the criteria for intravenous thrombolytic therapy received intravenous thrombolysis (alteplase; rt-PA) within $4.5 \mathrm{~h}$ of stroke onset after a CT scan, and an MRI examination immediately followed. If LVO (MCA or/and ICA) was found on MRA within $6 \mathrm{~h}$ of stroke onset, the thrombectomy was performed immediately. All patients were evaluated by MRI, including PWI and DWI. The patients included in the present study met the following criteria: (I) firstever acute stroke with MCA or/and ICA occlusion; (II) acute stroke patients $\leq 6$ hours of symptom onset; (III) pretreatment MRI with PWI, DWI and MR angiography (MRA); (IV) thrombectomy treatment and (V) followup MRI within 24 hours after thrombectomy therapy. The exclusion criteria were as follows: (I) cerebral hemorrhage, tumor or trauma detected by the CT scanner; (II) acute stroke patients with posterior cerebral artery (PCA) or vertebral artery (VA) occlusion; (III) any contraindication for MRI; (IV) any missing follow-up MRI after thrombectomy therapy; (V) refusal to undergo thrombectomy; and (VI) any MRI or digital subtraction angiography (DSA) that could not be evaluated due to a motion artifact (Figure 1).

Age, sex, hypertension, diabetes mellitus, hyperlipidemia, atrial fibrillation, homocysteine, and the National Institutes 


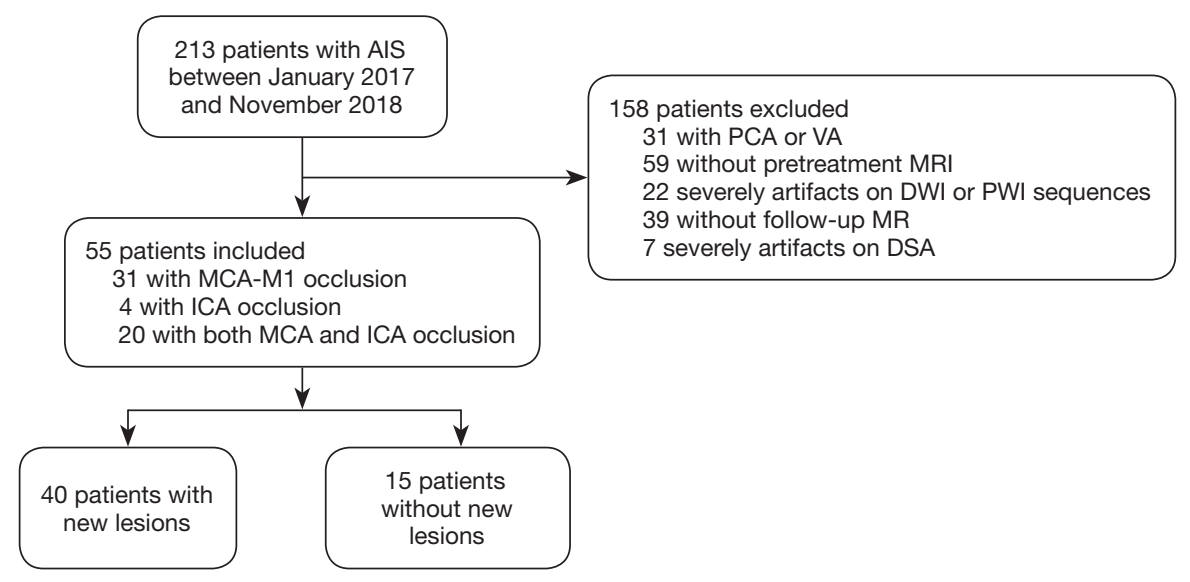

Figure 1 The flow chart in the study. AIS, acute ischemic stroke; PCA, posterior cerebral artery; VA, vertebral artery; MRI, magnetic resonance imaging; DWI, diffusion-weighted imaging; PWI, perfusion-weighted imaging; MR, magnetic resonance; DSA, digital subtraction angiography; ICA, internal carotid artery; MCA, middle cerebral artery.

of Health Stroke Scale (NIHSS) score at the admission hospital were collected. All patients had written informed consent by themselves or their family members prior to their participation in this study.

\section{MRI protocol}

Pretreatment and follow-up MRI scans were performed using a 3.0 Tesla MRI scanner (Ingenia, Philips Medical Systems) with an 8-channel receiver array head coil. The MRI protocol included fluid-attenuated inversion recovery (FLAIR) axis sequencing, DWI axial scanning, MRA and PWI. The scanning parameters were as follows: FLAIR [inverse recovery (IR) sequence, TR $7000 \mathrm{~ms}$, TE $120 \mathrm{~ms}$, acquisition matrix, 356 $\times 151$; field of view (FOV), $230 \mathrm{~mm}$ $\times 230 \mathrm{~mm}$; flip angle (FA), $90^{\circ}$; slices, 18 ; section thickness, $6 \mathrm{~mm}$; and intersection gap, $1.3 \mathrm{~mm}$ ]; and DWI [spin echo (SE) sequence, TR, 2,501 ms; TE, $98 \mathrm{~ms}$; acquisition matrix, 152×122; 3 directions; FOV, $230 \mathrm{~mm} \times 230 \mathrm{~mm}$; FA, $90^{\circ}$; slices, 18 ; section thickness, $6 \mathrm{~mm}$; and intersection gap, $1.3 \mathrm{~mm}$. DWI was obtained with $\mathrm{b}$ values of 0 and $\left.1,000 \mathrm{~s} / \mathrm{mm}^{2}\right]$. The 3D-MRA scans were obtained using the fast field echo (FFE) sequence with the following parameters: TR, $4.9 \mathrm{~ms}$; TE, $1.82 \mathrm{~ms}$; acquisition matrix, $528 \times 531$; FOV, $330 \mathrm{~mm} \times 330 \mathrm{~mm}$; and section thickness, $1.2 \mathrm{~mm}$. The imaging parameters for the DSC-PWI were as follows: TR, 1,620 ms; TE, $40 \mathrm{~ms}$; acquisition matrix, 96×93; >2 NEX; FOV, $224 \mathrm{~mm} \times 224 \mathrm{~mm}$; FA, 90% section thickness, $4 \mathrm{~mm}$; and duration $=88 \mathrm{~s}$.

\section{Image analysis}

The PWI data were analyzed using a Philips advanced workstation. The arterial input function (AIF) was selected by manually identifying the M2 segment of the MCA ipsilateral to the acute infarction. The CBF, CBV, TTP, and MTT maps were generated after circular singular value decomposition of the concentration-time curve. For each patient, a region of interest (ROI) was drawn in the core infarct areas (high signal tissue on DWI image), the at-risk hypoperfusion areas (hypoperfusion area with new lesions at follow-up MRI) and the other hypoperfusion areas. All ROIs were drawn on a TTP map, and the ROI volume encompassed the maximum abnormal perfusion area at the largest lesion level. Then, the relative CBF (rCBF), relative CBV (rCBV), MTT, and TTP values were automatically obtained. All parameters in the different areas were measured three times and averaged (by H.C with 3 years of experience board-certified radiologists). New lesions were evaluated based on DWI images before and after therapy, all new diffusion hyperintensities regardless of size and ADC value were considered. New lesions were defined as new DWI lesions which new high signal on followup DWI and low signal on follow-up ADC, and also not contiguous with initial abnormalities (Figure 2). Core infarct areas were defined as high signal on DWI and ADC values $<620 \times 10^{3} \mathrm{~mm}^{2} / \mathrm{s}$. the at-risk hypoperfusion areas were defined as the hypoperfusion areas on PWI before therapy corresponding to the new lesions. The other hypoperfusion areas were defined as the hypoperfusion areas on PWI before 

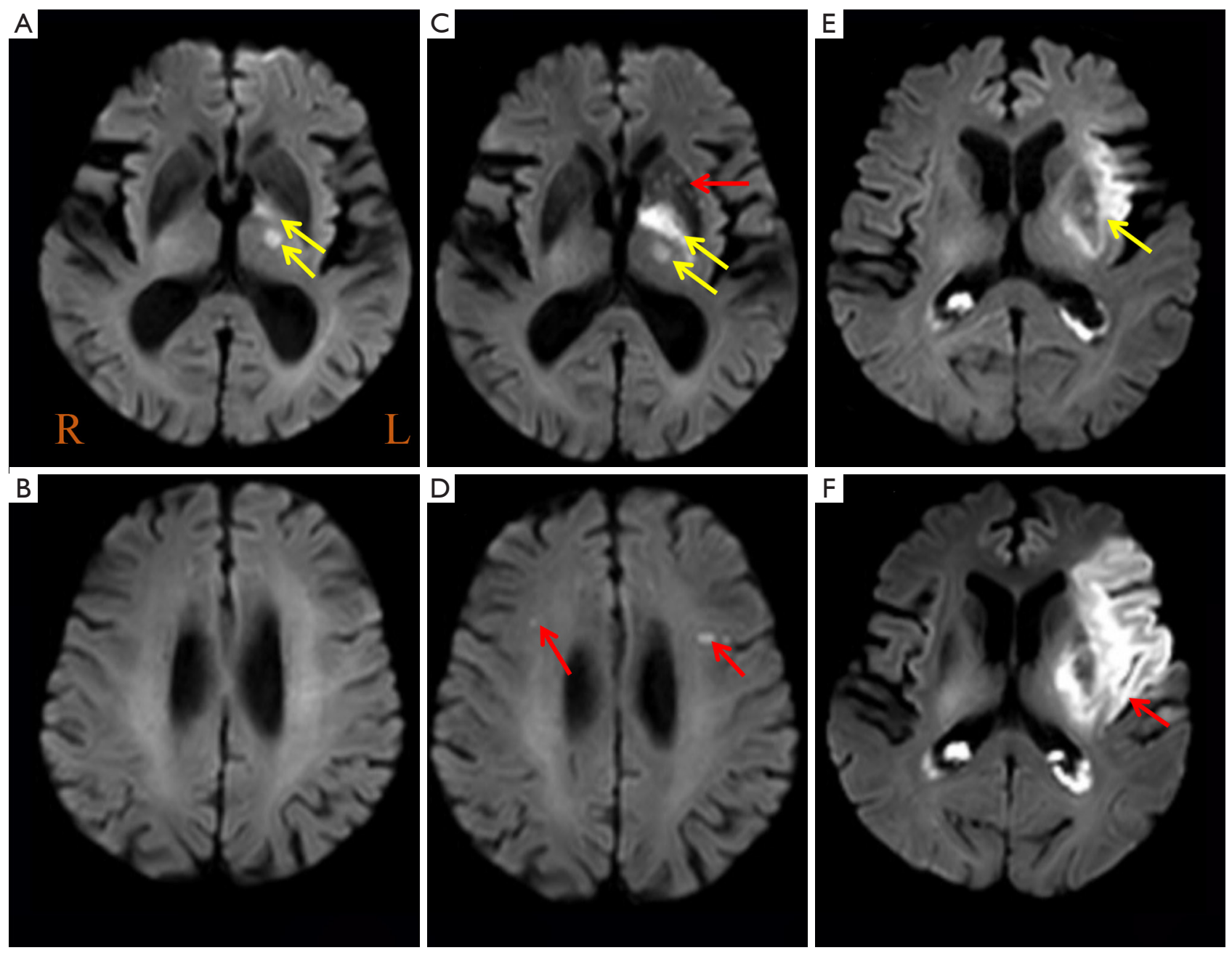

Figure 2 Examples of acute stroke patients with new lesions (A,B,C,D). (A,B) On the pretreatment DWI, there were multifocal infarcts in the left thalamus area (yellow arrows). (C,D) New ischemic lesions were observed at the follow-up DWI (red arrows). Examples of acute stroke patients without new lesions (E,F). (E) On the pretreatment DWI, there were local infarcts in the left basal ganglia and temporal lobe areas (yellow arrow). (F) The growth lesion on the follow-up DWI (red arrow) was continuous. Note that the left side corresponds to the right hemisphere. DWI, diffusion-weighted imaging.

therapy except new lesions and core infarct areas. Because there is no the at-risk hypoperfusion areas in patients without new lesions, the other hypoperfusion areas were defined as the hypoperfusion areas on PWI before therapy except core infarct areas. In cases of disagreements between the two board-certified radiologists reviewed about new lesions (H.C with 3 years of experience and M.P with 5 years of experience), the images were reviewed, and a consensus was established.

The thrombectomy of all patients was performed by two experienced interventional neuroradiologists (H.S with 13 years of experience; B. $Z$ with 7 years of experience) together, and they were blinded to the clinical information to assess the baseline angiography data of the endovascular therapy patients. The degree of reperfusion was evaluated with the Thrombolysis in Cerebral Infarction (TICI) grading system $(16)(0=$ complete occlusion to $3=$ complete revascularization). A good mTICI score was defined as mTICI $2 b-3$.

\section{Statistical analysis}

Continuous data are shown as the mean $\pm \mathrm{SD}$, whereas categorical variables are presented as absolute and relative frequencies. We analyzed the differences between groups 
Table 1 Comparison of patients with new lesions and without new lesions

\begin{tabular}{|c|c|c|c|c|}
\hline Variables & Patients with new lesions $(n=40)$ & Patients without new lesions $(n=15)$ & t value & $P$ value \\
\hline Age, years & $74.80 \pm 8.89$ & $66.00 \pm 9.86$ & 3.175 & $0.020^{*}$ \\
\hline Time from onset to admission, $\mathrm{h}$ & $2.75 \pm 1.76$ & $3.47 \pm 1.87$ & -1.323 & 0.192 \\
\hline Time from onset to first MRI, $h$ & $3.80 \pm 0.85$ & $3.15 \pm 1.04$ & -1.692 & 0.094 \\
\hline Smoking & $6(15.00 \%)$ & $2(13.33 \%)$ & 0.005 & 0.947 \\
\hline Alcohol drinking & $6(15.00 \%)$ & $0(0 \%)$ & 0.000 & 1.000 \\
\hline Diabetes mellitus & $22(55.00 \%)$ & $3(20.00 \%)$ & 5.390 & $0.020^{*}$ \\
\hline Hypertension & $33(82.50 \%)$ & $14(93.33 \%)$ & 0.343 & 0.558 \\
\hline Homocysteine & $5(12.50 \%)$ & $2(13.33 \%)$ & 0.000 & 1.000 \\
\hline NIHSS at admission & $13.20 \pm 6.08$ & $13.33 \pm 5.51$ & -0.074 & 0.941 \\
\hline $\mathrm{mTICI}(2 \mathrm{~b}-3)$ & 37 (92.50\%) & $14(93.33 \%)$ & 0.000 & 1.000 \\
\hline \multicolumn{5}{|l|}{ Location of occlusion } \\
\hline Middle cerebral artery-M1 & $21(52.50 \%)$ & $10(66.67 \%)$ & 0.890 & 0.345 \\
\hline Internal carotid artery & $2(5.00 \%)$ & $2(13.33 \%)$ & 0.227 & 0.633 \\
\hline Both & $17(42.50 \%)$ & $3(20.00 \%)$ & 2.387 & 0.208 \\
\hline
\end{tabular}

*, $\mathrm{P}<0.05$. NIHSS, National Institutes of Health Stroke Scale; mTICI, Modified Thrombolysis in Cerebral Ischemia.

using the chi-squared test for categorical variables and the independent sample $t$-test or Fisher's exact test for continuous variables. The differences between the core infarct areas and the at-risk areas, the core infarct areas and the other hypoperfusion areas, and the at-risk areas and the other hypoperfusion areas were analyzed by using paired $t$-tests. $\mathrm{P}<0.05$ was considered statistically significant. Logistic regression analysis of significantly associated variables $(\mathrm{P}<0.05)$ was used to identify factors predictive of new lesions. Binary logistic regression analysis used backward elimination to screen independent variables, and the odds ratio (OR) and 95\% confidential interval (CI) were obtained. Receiver operating characteristic (ROC) curve analysis was used to assess the predictive value of the independent variables $(\mathrm{P}<0.05)$ for the occurrence of new lesions in patients with acute stroke after thrombectomy. The differences of the predictive value from ROC analysis were evaluated according to DeLong et al. (17). All statistical analyses were conducted using commercially available software (SPSS for Windows, version 19.0; SPSS).

\section{Results}

During the study period, 213 patients underwent thrombectomy. Among these patients, one hundred and fifty-eight patients were excluded (patients with PCA or VA occlusion, $n=31$; no pretreatment MRI, $n=59$; severe artifacts on DWI or PWI, n=22; no follow-up MRI, n=39; severe artifacts on DSA, n=7) (Figure 1). Fifty-five patients were analyzed (Table 1); among these patients, forty patients (72.73\%) had new lesions on the follow-up MRI, and fifteen patients $(27.27 \%)$ did not have new lesions. The patients did not differ with regard to sex $(\mathrm{P}=0.655)$, time from onset to admission ( $\mathrm{P}=0.192)$, time from onset to first MRI ( $\mathrm{P}=0.094)$, time from onset to thrombectomy $(\mathrm{P}=0.986)$, hypertension $(\mathrm{P}=0.558)$, atrial fibrillation $(\mathrm{P}=0.134)$, hyperlipidemia $(\mathrm{P}=1.000)$, homocysteine $(\mathrm{P}=1.000)$, NIHSS score at admission $(\mathrm{P}=0.941)$, and mTICI score $(\mathrm{P}=1.000)$. The patients with new lesions were significantly older and more patients with new lesions had a history of diabetes mellitus than patients without new lesions $(\mathrm{P}=0.020$; 
Table 2 Comparison of CI, AR-H, TOH in patients with new lesions and without new lesions

\begin{tabular}{|c|c|c|c|c|}
\hline Parameter comparison & With new lesions & Without new lesions & t value & $P$ value \\
\hline \multicolumn{5}{|l|}{ rCBF } \\
\hline $\mathrm{Cl}$ vs. $\mathrm{Cl}$ & $103.07 \pm 81.50$ & $87.05 \pm 76.58$ & 0.660 & 0.512 \\
\hline TOH vs. TOH & $165.71 \pm 106.71$ & $181.44 \pm 107.43$ & -0.486 & 0.629 \\
\hline \multicolumn{5}{|l|}{ rCBV } \\
\hline AR-H vs. TO H & $24.14 \pm 37.30$ & $22.48 \pm 10.27$ & 0.170 & 0.866 \\
\hline TOH vs. TOH & $27.50 \pm 19.01$ & $22.48 \pm 10.27$ & 0.968 & 0.337 \\
\hline \multicolumn{5}{|l|}{ MTT } \\
\hline $\mathrm{Cl}$ vs. $\mathrm{Cl}$ & $12.22 \pm 3.51$ & $10.42 \pm 3.08$ & 1.750 & 0.086 \\
\hline $\mathrm{Cl}$ vs. $\mathrm{Cl}$ & $37.55 \pm 10.85$ & $38.60 \pm 12.65$ & -0.297 & 0.768 \\
\hline AR-H vs. TOH & $38.30 \pm 11.39$ & $28.67 \pm 6.06$ & 4.037 & $<0.0001^{*}$ \\
\hline $\mathrm{TOH}$ vs. $\mathrm{TOH}$ & $29.76 \pm 6.86$ & $28.67 \pm 6.06$ & 0.541 & 0.591 \\
\hline
\end{tabular}

*, $\mathrm{P}<0.05$. rCBF, relative cerebral blood flow (mL/100 g/min); rCBV, relative cerebral blood volume (mL/100 g); MTT, mean transit time (s); TTP, time to peak (s); Cl, the core infarct areas; AR-H, the at-risk hypoperfusion areas; TOH, the other hypoperfusion areas.

Table 3 Comparison of CI, AR-H, TOH in patients with new lesions

\begin{tabular}{|c|c|c|}
\hline Parameter comparison & T value & $P$ value \\
\hline \multicolumn{3}{|l|}{ rCBF } \\
\hline $\mathrm{Cl}$ vs. AR-H & -1.137 & 0.263 \\
\hline $\mathrm{Cl}$ vs. $\mathrm{TOH}$ & -4.035 & $<0.0001^{\star}$ \\
\hline AR-H vs. TOH & -0.610 & 0.319 \\
\hline \multicolumn{3}{|l|}{ rCBV } \\
\hline $\mathrm{Cl}$ vs. AR-H & -1.003 & 0.322 \\
\hline $\mathrm{Cl}$ vs. $\mathrm{TOH}$ & -3.916 & $<0.0001^{\star}$ \\
\hline $\mathrm{AR}-\mathrm{H}$ vs. TOH & -0.812 & 0.422 \\
\hline \multicolumn{3}{|l|}{ MTT } \\
\hline $\mathrm{Cl}$ vs. AR-H & 0.539 & 0.593 \\
\hline $\mathrm{Cl}$ vs. $\mathrm{TOH}$ & 6.041 & $<0.0001^{\star}$ \\
\hline AR-H vs. TOH & 5.933 & $<0.0001^{*}$ \\
\hline \multicolumn{3}{|l|}{ TTP } \\
\hline $\mathrm{Cl}$ vs. AR-H & -0.336 & 0.739 \\
\hline $\mathrm{Cl}$ vs. $\mathrm{TOH}$ & 4.828 & $<0.0001^{*}$ \\
\hline AR-H vs. TOH & 4.507 & $<0.0001^{\star}$ \\
\hline
\end{tabular}

*, $\mathrm{P}<0.05 . \mathrm{rCBF}$, relative cerebral blood flow $(\mathrm{mL} / 100 \mathrm{~g} / \mathrm{min})$; rCBV, relative cerebral blood volume $(\mathrm{mL} / 100 \mathrm{~g})$; MTT, mean transit time (s); TTP, time to peak (s); $\mathrm{Cl}$, the core infarct areas; $\mathrm{AR}-\mathrm{H}$, the at-risk hypoperfusion areas; $\mathrm{TOH}$, the other hypoperfusion areas.
$\mathrm{P}=0.020$, respectively). The artery occlusion in patients with new lesions was often located in the MCA-M1 (52.50\%) and both the MCA-M1 and ICA (42.50\%), while patients without new lesions often had occlusions located in the MCA-M1 (66.67\%); there was no significant difference in location of occlusion $(\mathrm{P}>0.05)$ (Table 1).

Table 2 showed the comparison of the core infarct areas, the at-risk hypoperfusion areas, and the other hypoperfusion areas in patients with new lesions and without new lesions. The MTT and TTP in the at-risk hypoperfusion areas with new lesions were significantly longer than in the hypoperfusion areas without new lesions $(11.95 \pm 3.29 \mathrm{vs}$. $8.02 \pm 2.08, \mathrm{P}<0.0001 ; 38.30 \pm 11.39 ; 28.67 \pm 6.06, \mathrm{P}<0.0001$, respectively). The PWI parameters were not significantly different between the core infarcts areas with new lesions and the core infarcts areas without lesions, the other hypoperfusion areas between patients with new lesions and without new lesions $(\mathrm{P}>0.05)$. In addition, we also compared the differences in PWI parameters between the core infarct areas, the at-risk hypoperfusion areas, and the other hypoperfusion areas in patients with new lesions (Table 3). All PWI parameters between the core infarct areas and the at-risk hypoperfusion areas were no significantly difference in patients with new lesions $(\mathrm{P}>0.05)$ while all 

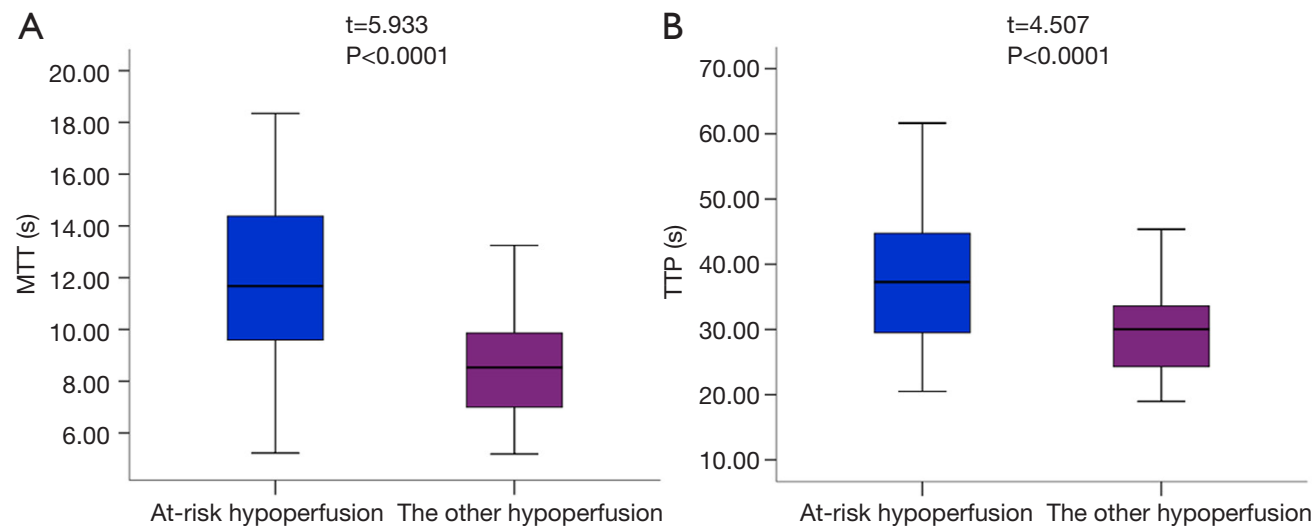

Figure 3 The MTT and TTP values in the at-risk hypoperfusion areas and the other hypoperfusion areas are displayed as a box plot. The MTT and TTP values in the at-risk hypoperfusion areas were significantly higher than those in the other hypoperfusion areas. MTT, mean transit time; TTP, time to peak.

PWI parameters between the core infarct and the other hypoperfusion areas were significantly different in patients with new lesions $(\mathrm{P}<0.05)$. Acute stroke patients with new lesions showed longer MTT in the at-risk hypoperfusion areas $(11.95 \pm 3.29)$ than in the other hypoperfusion areas $(8.68 \pm 2.08)$, and the TTP $(38.30 \pm 11.39)$ was also longer in the at-risk hypoperfusion areas than in the other hypoperfusion areas (29.76 \pm 6.86$)$ (Table 2; Figure 3), both of which were significantly different $(\mathrm{P}<0.0001$; $\mathrm{P}<0.0001$, respectively). Figure 4 showed the PWI images of acute stroke patients without new lesions. Figure 5 showed the PWI images of acute stroke patients with new lesions.

Logistic regression analysis demonstrated that MTT (OR, 0.623; 95\% CI, 0.455-0.851, $\mathrm{P}=0.003$ ) and TTP (OR, 0.776 ; 95\% CI, 0.584-0.942; $\mathrm{P}=0.016$ ) were independently associated with new lesions. We therefore investigated whether and at what threshold pathological MTT and TTP can predict new lesions using ROC analyses. The ROC analyses showed that the AUCs of MTT and TTP for predicting new lesions was 0.860 (95\% CI, 0.780-0.941) and 0.790 (95\% CI, 0.691-0.889), respectively (Figure 6). When the optimal cut-off value of MTT was 9.87 , the sensitivity and specificity for predicting new lesions were $70.00 \%$ and $87.50 \%$, respectively; the cut-off for TTP was 32.66 , and the sensitivity and specificity were $70.00 \%$ and $80.00 \%$, respectively. There was no difference of AUC curve between MTT and TTP $(\mathrm{z}=1.489 ; \mathrm{P}=0.1366)$.

\section{Discussion}

The results of the present study suggest that acute stroke patients had a high frequency of new lesions, and approximately $72.73 \%$ of the patients in this study had new lesions. It is interesting that patients with new lesions had longer MTTs and TTPs in the at-risk hypoperfusion areas than in the other hypoperfusion areas, and these differences were significant. The ROC analyses showed that the sensitivity and specificity of MTT for predicting new lesions were $70.00 \%$ and $87.50 \%$, respectively; the sensitivity and specificity of TTP were $70.00 \%$ and $80.00 \%$, respectively. PWI may be useful in monitoring the perfusion status and in predicting the early new lesions of acute stroke patients after thrombectomy.

PWI is a semiquantitative method for evaluating brain perfusion-microcirculation in the capillary network $(18,19)$ and can obtain various perfusion parameters, such as $\mathrm{CBF}$, CBV, TTP, MTT and time-to-maximum (Tmax). CBF, CBV, TTP and MTT maps could be generated using post processing workstation of MRI. While Tmax maps were generated using professional business software, such as RAPID software. In our study, all ROIs were drawn on a TTP map and then the other ROIs were automatically obtained, but cannot automatically obtained on Tmax due to different software. There are errors if drawing ROI of each area individually on Tmax maps using RAPID software. Besides, Wouters et al. showed that rTTP yields lesion volumes that are comparable to Tmax (20). Therefore, the parameters CBF, CBV, TTP and MTT not Tmax were analyzed. Yamada et al. demonstrated that selection of AIF from the peri-infarct artery is one of the techniques of choice to delineate the areas with true MTT prolongation in the presence of steno-occlusive disease (21). 

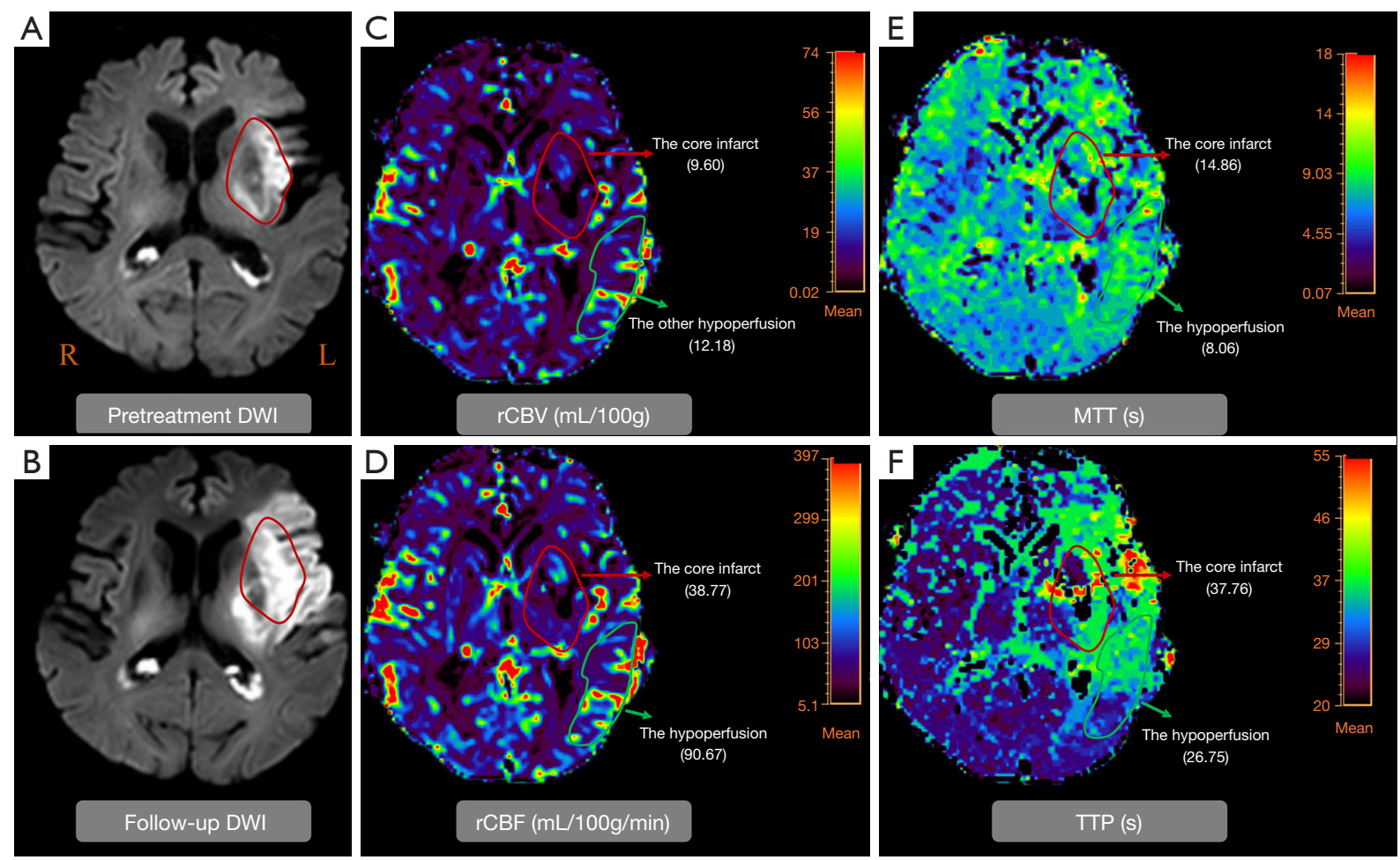

Figure 4 Illustrative case of an acute stroke without new lesions. The pretreatment DWI (A) showed hyperintense lesions in the left basal ganglia and temporal lobe areas. The lesions were continuously larger on the follow-up DWI than on the initial DWI (B). The red circle represented the core infarct region. $\mathrm{rCBV}(\mathrm{mL} / 100 \mathrm{~g})(\mathrm{C}), \mathrm{rCBF}(\mathrm{mL} / 100 \mathrm{~g} / \mathrm{min})(\mathrm{D}), \mathrm{MTT}(\mathrm{s})(\mathrm{E})$, and TTP(s) (F) are shown in the region of interest in the core infarct areas and hypoperfusion areas. Note that the left side corresponds to the right hemisphere; DWI, diffusionweighted imaging; rCBV, relative cerebral blood volume; rCBF, relative cerebral blood flow; MTT, mean transit time; TTP, time to peak.

In our study, MCA stenosis or occlusion was present in all acute stroke patients, so we selected the M2 segment of the ipsilateral MCA used for AIF to attain high sensitivity calculation techniques. In this study, the PWI parameters in the core infarct areas and the hypoperfusion areas were compared between patients with acute stroke who received thrombectomy. Regardless of if the patients had new lesions or did not have new lesions, the rCBF, rCBV, MTT and TTP were all significantly different between the core infarct areas and the hypoperfusion areas. These findings are in good agreement with those of a previous study (22-24). The core infarct is irreversibly infarcted tissue, while the hypoperfusion areas around the core infarct areas are salvageable brain tissue, which may develop into infarcted tissue (as new lesions) or recover after timely treatment (25-27). In our study, all patients within $6 \mathrm{~h}$ of the onset of ischemic symptoms were enrolled in order to prevent the heterogeneity caused by thrombectomy time. Besides, the incidence of new lesions is higher than the other study (1), the possible reason is that all patients in this study received thrombectomy. In addition, we found that patients with new lesions were more likely to have a history of diabetes mellitus than patients without new lesions. Poor glycemic control leads to endothelial dysfunction, coagulative activation, and platelet hyperreactivity (28). Patients with new lesions were significantly older than patients without new lesions. Some older patients may exhibit de novo lesions or incomplete clot dissolution due to various comorbidities and fragility as a result of their advanced age.

Thus far, the pathophysiology of these new lesions remains unclear. Vessel recanalization therapy possibly contributed to the occurrence of new lesions by incomplete clot dissolution and distal embolization $(6,7)$, or the new lesions may represent de novo lesions. In our study, we found that the mTICI score was not significantly different between stroke patients with new lesions and those without new lesions after thrombectomy. In addition, the strengths of our study were that we found stroke patients with new 

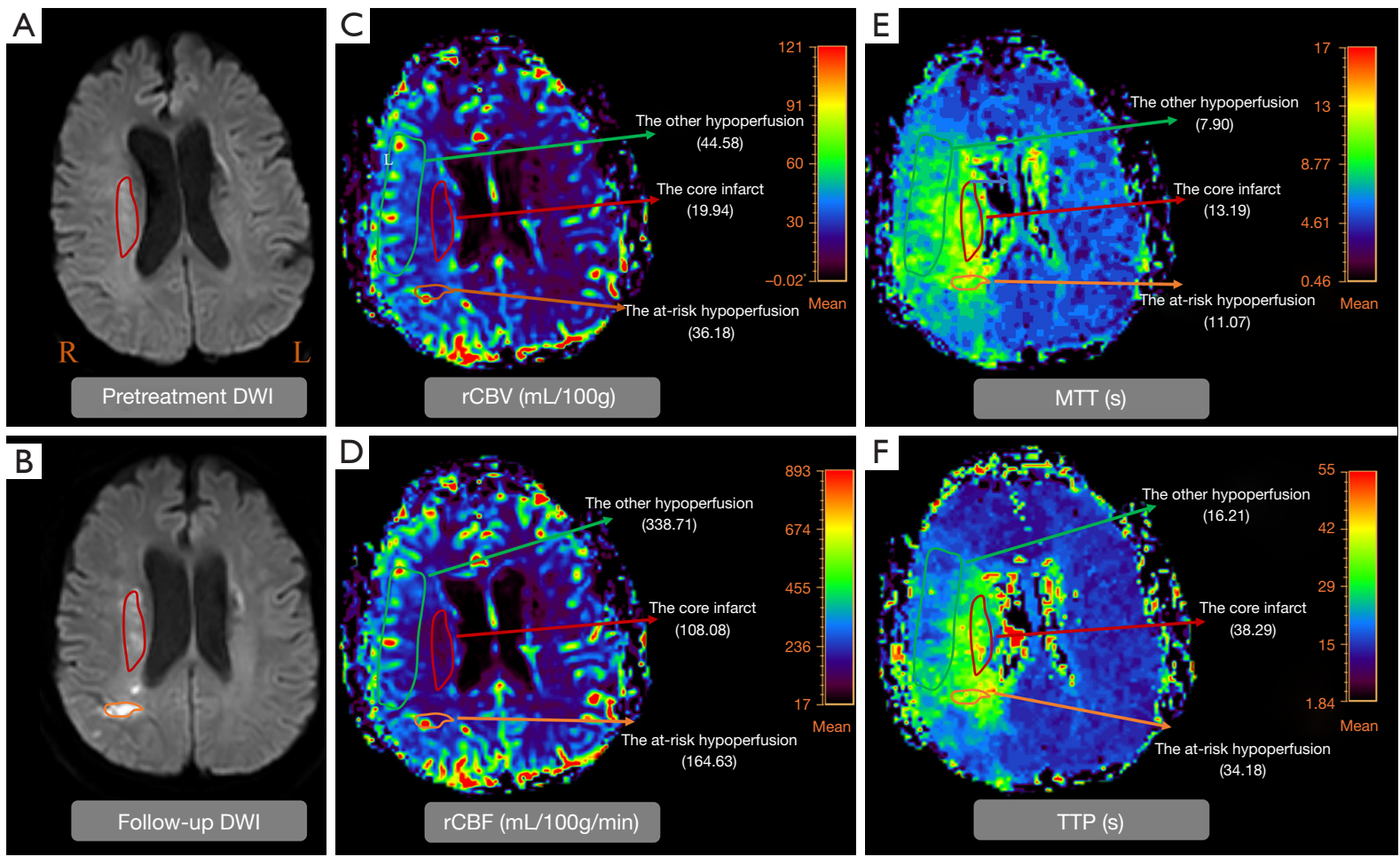

Figure 5 Illustrative case of an acute stroke with new lesions. The pretreatment DWI (A) showed hyperintense lesions in the right lateral ventricle area. The lesions were also visible in the right occipital lobe areas on the follow-up DWI (B). The red circle represented the core infarct region and the orange circle represented the at-risk hypoperfusion region. $\mathrm{rCBV}(\mathrm{mL} / 100 \mathrm{~g})(\mathrm{C}), \mathrm{rCBF}(\mathrm{mL} / 100 \mathrm{~g} / \mathrm{min})(\mathrm{D})$, MTT(s) (E), and TTP(s) (F) are shown in the region of interest in the core infarct areas, the at-risk hypoperfusion areas and the other hypoperfusion areas. (E,F) Showed that the MTT and TTP values in the at-risk hypoperfusion areas were longer than that in the other hypoperfusion areas. Note that the left side corresponds to the right hemisphere; DWI, diffusion-weighted imaging; rCBV, relative cerebral blood volume; rCBF, relative cerebral blood flow; MTT, mean transit time; TTP, time to peak.

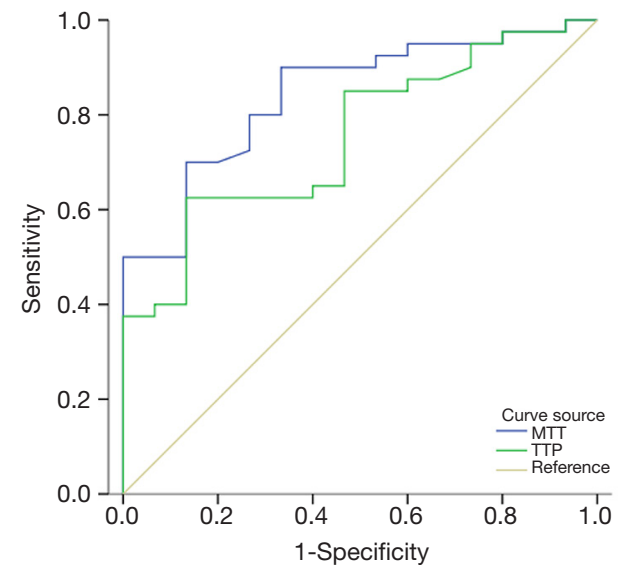

Figure 6 ROC curves showed MTT and TTP for predicting the occurrence of the new lesions (MTT: cut-off value: 9.87, sensitivity: $70.00 \%$, specificity: $87.50 \%$; TTP: cut-off value: 32.66 , sensitivity: $70.00 \%$, specificity: $80.00 \%$, respectively). ROC, receiver operating characteristic; MTT, mean transit time; TTP, time to peak. lesions to have different hypoperfusion degrees between the new lesions (namely, the at-risk hypoperfusion areas) and the other hypoperfusion areas. The occurrence of new lesions within the hypoperfusion areas may be related to thromboembolic mechanisms (11). Caplan et al. (29) hypothesized that impaired washout is an important mechanism of brain infarction in patients with cerebral hypoperfusion. Hypoperfusion enhances thrombus formation, promotes embolization of fresh thrombi and limits the ability of the blood stream to clear or wash out emboli $(29,30)$. Therefore, the new lesions in these patients are more likely to represent the natural evolution of the ischemic process and "completion" of the infarct within the territory of the penumbral deficit.

To further explore which patients will develop new lesions, we compared the differences in PWI parameters between the at-risk hypoperfusion and other hypoperfusion areas. We found that the $\mathrm{rCBF}$ and $\mathrm{rCBV}$ had no significant 
differences between in the at-risk hypoperfusion areas and the other hypoperfusion areas, which was similar to a previous study (31). Akazawa et al. demonstrated that the rCBF of "Growth" and "Reversed" areas had substantial overlap, which hampered the delineation of areas that would become infarcted (31). Their study showed that it is difficult to use MRI to differentiate between penumbra that will survive and that which will not. Arenillas et al. reported that lower $\mathrm{rCBV}$ on $\mathrm{CT}$ perfusion can predict a higher infarct growth in the endovascular treatment patients (32). While the strength of this study was the MTT and TTP in the at-risk hypoperfusion areas were longer than in the other hypoperfusion areas, and there were significant differences, which is different from the results of Bang et al. (11). The possible reasons are different evaluation method, their studies evaluated the association between new lesions on day 7 and Tmax hypoperfusion severity. In our study, we used the MTT and TTP hypoperfusion severity to predict the new lesions. The MTT is used to predict vulnerable brain tissue that may evolve from the infarction. TTP describes the time it takes $\mathrm{CBF}$ to reach the highest value at the target tissue location (33). Singh et al. presented a method to distinguish normal tissue and affected areas by texture analysis of CT images, and suggested that texture analysis could be a useful tool to help neurologists in the early detection of ischemic stroke with the accuracy of the algorithm is $93.3 \%$ (34). Arsava et al. assessed the utility of recurrence risk estimator (RRE) for predicting 7-day stroke in patients with transient symptoms with infarction (TSI) by using ROC analysis (35). They found that sensitivity and specificity of an RRE score of $\geq 2$ for predicting 7-day stroke risk were $87 \%$ and $73 \%$ respectively with the AUC was 0.85 (95\% CI, 0.78-0.92). In our study, we found that MTT and TTP were independently associated with new lesions using logistic regression analysis. We probed the predictive value of the pretreatment MTT and TTP by ROC analysis and found that the sensitivity and specificity of MTT for predicting new lesions were $70.00 \%$ and $87.50 \%$, respectively; the sensitivity and specificity of TTP were $70.00 \%$ and $80.00 \%$, respectively. Our results indicate that MTT and TTP might be predictors of new lesions.

This study has several limitations. First, this study had a small sample size that may subject our findings to sampling bias. This study enrolled a large number of patients from the beginning, but many heterogeneous cases were excluded after rigorous screening. Despite the small number of cases, we still found that MTT and TTP may have predictive value for new lesions. Our future study will confirm these results or possibly reveal other meaningful findings by expanding the sample size. Second, due to the small sample size, we did not divide it into train data and test data for determining the cut-off of TTP and MTT. Our future work will focus on validating these results with bigger cohort using train and test data. Third, the new lesions were not divided into new local lesions and new distal lesions because of the small sample size and thus, we cannot distinguish new lesions arising from de novo lesions or incomplete clot dissolution. However, all the patients underwent thrombectomy, and there was no significant difference between patients with new lesions and those without new lesions. Therefore, our study still has some value in predicting new lesions. Four, the parameter of $T_{\max }>6 \mathrm{~s}$ maybe more suitable for predicting new lesions than TTP and MTT, while the parameter of $T_{\max }>6 \mathrm{~s}$ can only be obtained by special software processing, which needs extra purchase and few hospitals purchased. Therefore, in this study, we used TTP and other easily available parameters to predict new lesions. Finally, this was a single-center trial. Thus, the results of this study cannot be generalized to whole population of stroke patients. Multicenter trials with larger cohorts and different study populations are warranted.

\section{Conclusions}

In summary, new lesions occurred in acute stroke patients at a high frequency. In patients with new lesions, the MTT and TTP in the at-risk hypoperfusion areas were significantly longer than in the other hypoperfusion areas. MTT and TTP may be useful in predicting the occurrence of early new lesions in acute stroke patients after thrombectomy.

\section{Acknowledgments}

Funding: This work was supported by the Natural Science Foundation of Jiangsu Province (No. BK20201118) and Jiangsu Provincial Special Program of Medical Science (No. BE2017614).

\section{Footnote}

Conflicts of Interest: All authors have completed the ICMJE uniform disclosure form (available at http://dx.doi. org/10.21037/qims-21-1). The authors have no conflicts of interest to declare. 
Ethical Statement: The authors are accountable for all aspects of the work in ensuring that questions related to the accuracy or integrity of any part of the work are appropriately investigated and resolved. The study was conducted in accordance with the Declaration of Helsinki (as revised in 2013). The Institutional Review Board approved the current study of Nanjing Medical University. Written informed consent was obtained from all participants before they participated in the study protocol.

Open Access Statement: This is an Open Access article distributed in accordance with the Creative Commons Attribution-NonCommercial-NoDerivs 4.0 International License (CC BY-NC-ND 4.0), which permits the noncommercial replication and distribution of the article with the strict proviso that no changes or edits are made and the original work is properly cited (including links to both the formal publication through the relevant DOI and the license). See: https://creativecommons.org/licenses/by-nc-nd/4.0/.

\section{References}

1. Lee J, Inoue M, Mlynash M, Mann SK, Cereda CW, Ke M, Albers GW, Olivot JM. MR perfusion lesions after TIA or minor stroke are associated with new infarction at 7 days. Neurology 2017;88:2254-9.

2. Gladstone DJ, Kapral MK, Fang J, Laupacis A, Tu JV. Management and outcomes of transient ischemic attacks in Ontario. CMAJ 2004;170:1099-104.

3. Kang DW, Latour LL, Chalela JA, Dambrosia J, Warach S. Early ischemic lesion recurrence within a week after acute ischemic stroke. Ann Neurol 2003;54:66-74.

4. Lee EJ, Kang DW, Warach S. Silent New Brain Lesions: Innocent Bystander or Guilty Party? J Stroke 2016;18:38-49.

5. Kang DW, Han MK, Kim HJ, Sohn H, Kim BJ, Kwon SU, Kim JS, Warach S. Silent new ischemic lesions after index stroke and the risk of future clinical recurrent stroke. Neurology 2016;86:277-85.

6. Asdaghi N, Hameed B, Saini M, Jeerakathil T, Emery D, Butcher K. Acute perfusion and diffusion abnormalities predict early new MRI lesions 1 week after minor stroke and transient ischemic attack. Stroke 2011;42:2191-5.

7. Usnich T, Albach FN, Brunecker P, Fiebach JB, Nolte $\mathrm{CH}$. Incidence of new diffusion-weighted imaging lesions outside the area of initial hypoperfusion within 1 week after acute ischemic stroke. Stroke 2012;43:2654-8.

8. Nolte CH, Albach FN, Heuschmann PU, Brunecker P,
Villringer K, Endres M, Fiebach JB. Silent new DWI lesions within the first week after stroke. Cerebrovasc Dis 2012;33:248-54.

9. Kang DW, Yoo SH, Chun S, Kwon KY, Kwon SU, Koh JY, Kim JS. Inflammatory and hemostatic biomarkers associated with early recurrent ischemic lesions in acute ischemic stroke. Stroke 2009;40:1653-8.

10. Kang DW, Lattimore SU, Latour LL, Warach S. Silent ischemic lesion recurrence on magnetic resonance imaging predicts subsequent clinical vascular events. Arch Neurol 2006;63:1730-3.

11. Bang OY, Kim GM, Chung CS, Kim SJ, Kim KH, Jeon P, Saver JL, Liebeskind DS, Lee KH. Differential pathophysiological mechanisms of stroke evolution between new lesions and lesion growth: perfusionweighted imaging study. Cerebrovasc Dis 2010;29:328-35.

12. Braemswig TB, Usnich T, Albach FN, Brunecker P, Grittner U, Scheitz JF, Fiebach JB, Nolte CH. Early new diffusion-weighted imaging lesions appear more often in stroke patients with a multiple territory lesion pattern. Stroke 2013;44:2200-4.

13. Jeon SB, Song HS, Kim BJ, Kim HJ, Kang DW, Kim JS, Kwon SU. Biochemical aspirin resistance and recurrent lesions in patients with acute ischemic stroke. Eur Neurol 2010;64:51-7.

14. Jeong TD, Kim SM, Kim HJ, Lee W, Kwon SU, Min WK, Kang DW, Chun S. CYP2C19 genotype and early ischemic lesion recurrence in stroke patients treated with clopidogrel. J Stroke Cerebrovasc Dis 2015;24:440-6.

15. Mohan KM, Wolfe CD, Rudd AG, Heuschmann PU, Kolominsky-Rabas PL, Grieve AP. Risk and cumulative risk of stroke recurrence: a systematic review and metaanalysis. Stroke 2011;42:1489-94.

16. Zaidat OO, Lazzaro MA, Liebeskind DS, Janjua N, Wechsler L, Nogueira RG, Edgell RC, Kalia JS, Badruddin A, English J, Yavagal D, Kirmani JF, Alexandrov $\mathrm{AV}$, Khatri P. Revascularization grading in endovascular acute ischemic stroke therapy. Neurology 2012;79:S110-6.

17. DeLong ER, DeLong DM, Clarke-Pearson DL. Comparing the areas under two or more correlated receiver operating characteristic curves: a nonparametric approach. Biometrics 1988;44:837-45.

18. Tatlisumak T, Strbian D, Abo Ramadan U, Li F. The role of diffusion- and perfusion-weighted magnetic resonance imaging in drug development for ischemic stroke: from laboratory to clinics. Curr Vasc Pharmacol 2004;2:343-55.

19. Peng JW, Liu Y, Meng G, Zhang JY, Yu LF. Effects of salvianolic acid on cerebral perfusion in patients after acute 
stroke: A single-center randomized controlled trial. Exp Ther Med 2018;16:2600-14.

20. Wouters A, Christensen S, Straka M, Mlynash M, Liggins J, Bammer R, Thijs V, Lemmens R, Albers GW, Lansberg MG. A Comparison of Relative Time to Peak and Tmax for Mismatch-Based Patient Selection. Front Neurol 2017;8:539.

21. Yamada K, Wu O, Gonzalez RG, Bakker D, Østergaard L, Copen WA, Weisskoff RM, Rosen BR, Yagi K, Nishimura T. Magnetic resonance perfusion-weighted imaging of acute cerebral infarction: effect of the calculation methods and underlying vasculopathy. Stroke 2002;33:87-94.

22. Song SS. Advanced imaging in acute ischemic stroke. Semin Neurol 2013;33:436-40.

23. Tong E, Sugrue L, Wintermark M. Understanding the Neurophysiology and Quantification of Brain Perfusion. Top Magn Reson Imaging 2017;26:57-65.

24. Luo S, Yang L, Wang L. Comparison of susceptibilityweighted and perfusion-weighted magnetic resonance imaging in the detection of penumbra in acute ischemic stroke. J Neuroradiol 2015;42:255-60.

25. Chen C, Parsons MW, Clapham M, Oldmeadow C, Levi CR, Lin L, Cheng X, Lou M, Kleinig TJ, Butcher KS, Dong Q, Bivard A. Influence of Penumbral Reperfusion on Clinical Outcome Depends on Baseline Ischemic Core Volume. Stroke 2017;48:2739-45.

26. Chung JW, Kim JY, Park HK, Kim BJ, Han MK, Lee J, Choi KH, Kim JT, Jung C, Kim JH, Kwon OK, Oh CW, Lee J, Bae HJ. Impact of the Penumbral Pattern on Clinical Outcome in Patients with Successful Endovascular Revascularization. J Stroke Cerebrovasc Dis 2017;26:360-7.

27. Regenhardt RW, Das AS, Stapleton CJ, Chandra RV,

Cite this article as: Jiang L, Ai Z, Geng W, Chen H, Zhao B, Su H, Yin X, Chen YC. Predictive value of perfusion weighted imaging for early new lesions after stroke patients receive endovascular treatment. Quant Imaging Med Surg 2021;11(8):3643-3654. doi: 10.21037/qims-21-1
Rabinov JD, Patel AB, Hirsch JA, Leslie-Mazwi TM. Blood Pressure and Penumbral Sustenance in Stroke from Large Vessel Occlusion. Front Neurol 2017;8:317.

28. Rivas Rios JR, Franchi F, Rollini F, Angiolillo DJ. Diabetes and antiplatelet therapy: from bench to bedside. Cardiovasc Diagn Ther 2018;8:594-609.

29. Caplan LR, Hennerici M. Impaired clearance of emboli (washout) is an important link between hypoperfusion, embolism, and ischemic stroke. Arch Neurol 1998;55:1475-82.

30. Sedlaczek O, Caplan L, Hennerici M. Impaired washout-embolism and ischemic stroke: further examples and proof of concept. Cerebrovasc Dis 2005;19:396-401.

31. Akazawa K, Yamada K, Matsushima S, Kubota T, Nagakane Y, Kuriyama N, Nakagawa M, Nishimura T. Is it possible to define salvageable ischemic penumbra using semiquantitative rCBF levels derived from MR perfusionweighted imaging? Neuroradiology 2008;50:939.

32. Arenillas JF, Cortijo E, García-Bermejo P, Levy EI, Jahan R, Liebeskind D, Goyal M, Saver JL, Albers GW. Relative cerebral blood volume is associated with collateral status and infarct growth in stroke patients in SWIFT PRIME. J Cereb Blood Flow Metab 2018;38:1839-47.

33. Copen WA, Schaefer PW, Wu O. MR perfusion imaging in acute ischemic stroke. Neuroimaging Clin N Am 2011;21:259-83, $\mathrm{x}$.

34. Singh M, Garg V, Bhat P. Early Detection of Stroke using Texture Analysis. Indian Journal of Forensic Medicine \& Toxicology 2019;13:49-52.

35. Arsava EM, Furie KL, Schwamm LH, Sorensen AG, Ay H. Prediction of early stroke risk in transient symptoms with infarction: relevance to the new tissue-based definition. Stroke 2011;42:2186-90. 